\title{
Eine Art soziale Isolation
}

\section{Rouven Porz}

Prof. Dr., Medizinethik und ärztliche Weiterbildung, Insel Gruppe, Inselspital Bern

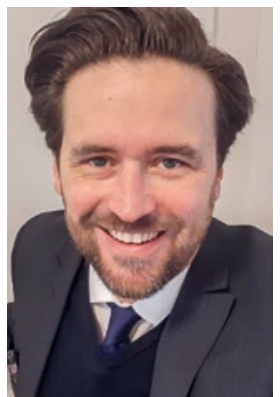

«Eigentlich fühle ich mich total isoliert und allein.» Das war keine Aussage eines Patienten, sondern vielmehr eine Antwort einer meiner Medizinstudentinnen. Dieses Frühjahr hatte ich sie im Ethikunterricht in einem kleinen Zoom-Gruppenunterricht danach gefragt, wie es ihnen denn mit der virtuellen Lehre eigentlich persönlich ginge.

Zuerst betroffenes Schweigen, dann: «Ich komme gar nicht mehr aus meiner Wohnung raus, ich sitze jeden Tag sechs bis acht Stunden vor dem Bildschirm: Podcasts, Zoom-Vorlesungen, Online-Plattformen, ich kann nicht mehr", so die eine Studentin, die anderen Studierenden nickten dazu in ihre Kameras. Eine andere erzählte: «Ich musste zum Arzt gehen, ich hatte Angst, dass ich verrückt werde. Ich habe gar keine Freunde mehr getroffen, ich konnte keinen Sport mehr machen, nur Uni, nur online.» Irgendwie haben mich diese offenen Bekenntnisse ziemlich berührt.

\section{Wir dürfen die soziale Komponente unseres} Unterrichts nie mehr als gegeben hinnehmen. Wir müssen sie immer neu kreieren.

Natürlich, die Pandemie hat sehr viele Themen aufgeworfen, die uns berührt haben. Oftmals an sich scheinbar triviale Themen, alte Menschen in Pflegeheimen, sozial schwache Klassen ohne Zugang zu medizinischer Versorgung, Impfgegner, Demonstrationen, Politikverdruss und so weiter und so fort. Aber allen diesen Themen wurde mit dem Brennglas der Pandemie plötzlich ein Mehr an ethischer Dramatik zugefügt - so auch, zumindest meiner Meinung nach, dieser neuen Einsamkeit meiner Studentinnen und Studenten.

Grund genug also, über die Lehre an Universitäten wieder neu nachzudenken (und Ähnliches gilt sicherlich auch für die meisten Schulen, Fachhochschulen etc.). Ich möchte mich dazu nur auf zwei Punkte konzentrieren, erstens, die offensichtlich nun neue Bedeutung der Lehre, und zweitens, den sozialen Aspekt der Lehre.

1) Die neue Bedeutung der Lehre: Wir wissen es ja eigentlich alle. Wenn man an einer Universität Karriere machen will, dann zählen vor allem Forschungsgelder und viele Publikationen. Die Lehre war (oder ist) meistens ein Nebenprodukt, das man irgendwie erfüllen muss, aber das in einer echten akademischen Karriere nicht so richtig wichtig ist. Man redet kaum darüber. Das Unterrichten war irgendwie selbstverständlich. Du tust es, aber du machst nicht viel Aufhebens darum, du wirst auch kaum von der Fakultät danach gefragt. Und das war jetzt plötzlich anders. Plötzlich war die Lehre wichtig. In der Corona-Krise musste man jetzt über die Lehre reden, wer macht was, wer macht es wie, und vor allem, wie schafft man gute Lehre "online». Plötzlich bekamen wir E-Mails von der Hochschulleitung zur Lehre, wir bekamen auch Tipps und wurden sogar gecoacht (zum Online-Unterrichten). Erstes Fazit: Durch die Krise wurde die Lehre plötzlich zu einem wichtigen Thema. Schade, dass es dazu der Krise bedurfte.

2) Der soziale Aspekt der Lehre: Ganz offensichtlich haben wir bislang viel zu wenig über die soziale Bedeutung der Lehre nachgedacht. Ich zumindest hatte das nie bedacht. Aber nachdem unsere Studierenden monatelang mehr oder weniger allein zu Hause gesessen waren, da realisierten wir Dozierenden plötzlich, inwiefern diese Einsamkeit bei vielen zu Lebenskrisen und Depressionen führte. Eine Art soziale Isolation. Eine Nebenwirkung der virtuellen Lehre sozusagen. Zur Uni gehen, im Hörsaal sitzen, Smalltalk mit anderen Kommilitoninnen und Kommilitonen, genau diese starke gesellschaftliche Komponente war mir nie bewusst, war ja auch normal, vorher hat es in der Geschichte der Menschheit (meines Wissens) noch nie virtuellen Unterricht gegeben. Aber was lernen wir jetzt daraus? Ich denke, wir lernen, dass wir die soziale Komponente unseres Unterrichts nie mehr als gegeben hinnehmen dürfen. Wir müssen sie immer neu kreieren, und das ist im Live-Kontakt viel einfacher. Wenn man somit über die positiven und negativen Auswirkungen der Covid-19-Pandemie für die Lehre nachdenkt, dann müssen wir uns eigentlich zugestehen, dass wir langfristig vor allem Positives gelernt haben. Wir müssen wissen, wie Online-Unterricht gut funktioniert, aber wir müssen uns auch immer eingestehen, wie wichtig die Lehre ist, und dass man alleine mit Forschungsgeldern und Publikationen noch keine interessierten, selbstkritischen, neugierigen und wissbegierigen Ärztinnen und Ärzte der Zukunft heranziehen kann - mit guter Lehre aber schon. 